\title{
SUNSPOT SEISMOLOGY THEORY
}

\author{
JOSEPH M. DAVILA \\ Laboratory for Astronomy and Solar Physics \\ NASA-Goddard Space Flight Center \\ Greenbelt, $M D 20771$
}

\begin{abstract}
Recent observations have demonstrated that sunspots can absorb significant p-mode wave power, and that this absorption has a well defined wavelength dependence. These observations open, for the first time, the potential to deduce semiempirically the subsurface structure of sunspots and active regions. Several physical mechanisms have been proposed to explain the absorption. In this paper, the proposed mechanisms are reviewed and their viability in view of the current knowledge of the scattering process is discussed.
\end{abstract}

\section{Introduction}

Below the solar photosphere waves are energetically unimportant. Most of the energy is carried by the convective or radiative flux. Sub-photospheric waves can however serve as powerful probes of the internal structure of the sun. Most of these studies have concentrated on the large scale properties of the solar interior. Recently however observations of the scattering of solar p-modes from sunspots have been obtained.

During the first half of this century physicists were able to deduce the structure of the atomic nucleus by observing the scattering of electromagnetic radiation. One might expect, by analogy, that the process of scattering of p-modes by sunspots will ultimately yield significant new insights into the subsurface structure of solar active regions, and ultimately to new ideas regarding the formation of active regions, sunspot structure and the influence of the dynamo on the solar activity cycle.

\section{Observations}

Previous attempts to use oscillations as a probe of sunspot structure have concentrated on velocities measured within the sunspots. These observations enabled a test of the hypothesis that the magnetic field of the spot acts as a selective filter of external wave energy (Thomas et al., 1982). There is also an apparent shift in wavenumbers in the spot relative to the surrounding photosphere, possibly related to the difference between

E. R. Priest and V. Krishan (eds.), Basic Plasma Processes on the Sun, 149-157.

(c) 1990 IAU. Printed in the Netherlands. 
sunspot magnetoacoustic speed and sound speed in the surrounding atmosphere (Abdelatif et al., 1986).

More recent work has taken a different point of view. Braun, Duvall and LaBonte (1987) have studied how the presence of the sunspot affects externally impinging waves, much as one would study the structure of an atom by bombarding it with electromagnetic radiation and observing the scattered radiation field. The high-degree acoustic oscillations are vertically trapped in a spherical shell with the surface as the upper boundary and the lower boundary depending on the frequency and horizontal wavenumber in a known way. It is likely that the high-degree waves are not global modes, that is, they do not remain coherent while travelling over a circumference to interfere with themselves. So locally they can be considered as horizontally travelling, vertically trapped waves. The waves are normally observed by their vertical motions near the surface, which we see as Doppler shifts of spectrum lines. In the classical analysis, waves in one fixed direction are isolated by spatial filtering of two-dimensional Doppler shift images and then a two-dimensional power spectrum isolates the behavior in horizontal wavenumber and temporal frequency. The two independent quadrants of this power spectrum correspond to waves travelling in opposite directions on the solar surface.

Braun, Duvall and LaBonte (1987) replaced the classical analysis by one in a cylindrical coordinate system centered on the sunspot. Radially propagating waves are isolated by a Hankel function decomposition from which the sunspot itself is excluded. The resulting two quadrants of the power spectrum correspond to inward and outward propagating waves. It is immediately obvious from one of these spectra that there is a large excess of power in the incoming waves over the outgoing waves. This excess is interpreted as being due to absorption by the sunspot. The absorption appears to be a function only of the horizontal wavenumber $k$ and not of the frequency $\omega$ or the phase velocity $\omega / k$ which would be expected for a purely depth dependent effect (Duvall, 1982). The absorption is small at low $k$ and increases to a maximum of $50 \%$ absorption at $k \sim 1 \mathrm{Mm}^{-1}$ and remains at that level to the maximum observed wavenumber $k=1.5 \mathrm{Mm}^{-1}$. This result was found for spots with $10 \mathrm{Mm}$ umbral diameter. For a smaller spot a reduced magnitude of absorption was found (Braun et al., 1988).

\section{Qualitative Discussion of the Scattering Process}

What is the physical nature of the mechanism responsible for this absorption? There are two possibilities, both illustrated in figure. The incoming wave flux, $P_{\text {in }}$ is greater than the outgoing power, $P_{\text {out }}$. The difference between these two quantities is defined as the absorbed power. The excess energy could be converted into heat within the sunspot by dissipative (irreversible) processes. The mechanism most frequently suggested is resonance absorption, a process which has been well studied in the context of heating coronal loops. It is also possible that the incoming sound wave shakes the magnetic field of the sunspot flux tube, and energy which is originally in sound waves is transferred into magnetic wave modes propagating more or less parallel to the magnetic field of the sunspot flux tube. If these waves can then leak from the system, say upward through the chromosphere or downward through the base of the sunspot (the flux is denoted as $S_{\text {mag }}$ 


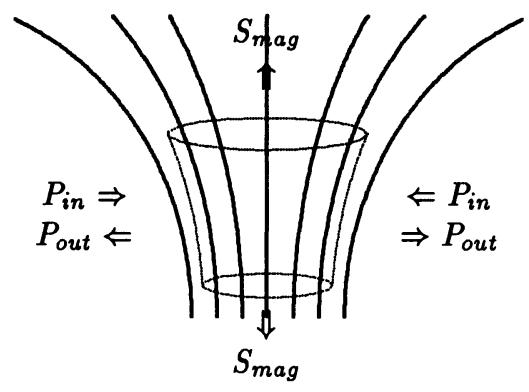

Figure 1: Physical processes which can result in the absorption of p-modes. The net power absorbed $P_{\text {in }}-P_{\text {out }}$ can be converted into magnetic modes which then propagate out through one of the ends of the sunspot flux tube, $S_{m a g}$, or the energy can be dissipated in a thin hollow cylindrical region.

in figure 1), one could explain the observed absorption by a completely non-dissipative process involving mode conversion in the field of the sunspot. Finally, it should be noted that these processes are not mutually exclusive. It is indeed possible that both of these basic mechanisms, i.e. dissipation and mode conversion, are responsible for the absorption seen in a real spot. At this point there is no observational way to distinguish between these possibilities.

From relatively simple considerations, however, one can make some educated guesses about what might be learned about the structure of sunspots by comparing theory and observational descriptions of the scattering process. For example if the magnetic field of the sunspot is converging with depth, as shown in figure $2 a$, one can see that the propagation direction of the outgoing p-modes is on the average directed more upward toward the solar surface than for the incoming waves. If on the other hand, the magnetic field diverges with depth, as shown in figure $2 b$, the average propagation direction of the p-modes is directed more downward toward the interior of the sun. It seems reasonable to hope that these two situations could be distinguished observationally once models are available to predict the magnitude and exact signature of the effect.

It seems unreasonable to imagine that a sunspot would remain as a vertical monolithic structure in the solar atmosphere. It is likely to be displaced from its vertical position by convective motions in the surrounding fluid and/or by magnetic buoyancy. By considering the simple structure shown in figure 2c, it is clear that the scattering of sound from such a structure would not be axisymmetric. The p-mode flux on one side of the spot is reflected upward on the average, and the flux on the other side is reflected downward. Asymmetries in the scattering rate have been observed. However it seems that they can be explained primarily by the "impact parameter" effect. (Braun et al., 1988) Additional modeling must be completed before one can hope to separate any additional asymmetry due to a non-vertical sunspot flux-tube from the asymmetry caused by this basic mechanism.

Finally, as indicated in figure 2d, one might hope to determine whether the sunspot 


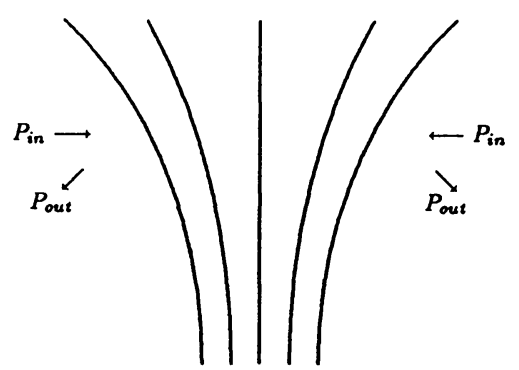

(a)

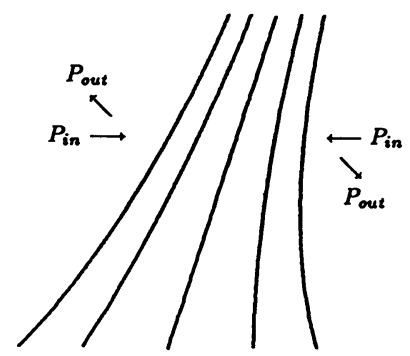

(c)

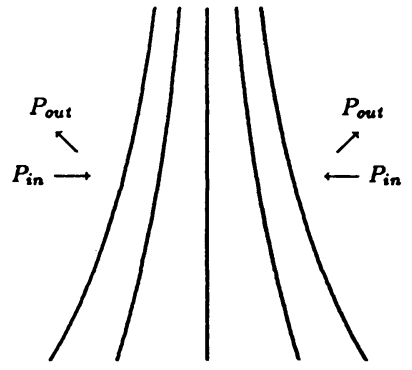

(b)

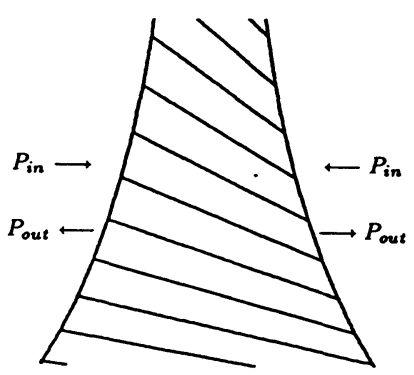

(d)

Figure 2: The geometry of the subsurface sunspot flux tube can affect the amplitude of the scattered wave observed at the surface. The panels represent diverging flux tubes (a), a converging flux tube (b), a non-vertical sunspot (c) and a twisted flux tube (d). Potential effects of these different configurations are discussed in the text.

flux tube carries a significant current, i.e. whether the flux tube is twisted. One might suspect that the presence of twist could significantly stiffen the flux tube and thereby change the predicted amplitude for waves reflected from the spot interface. Alternately as discussed below the presence of twist seems to be necessary for the efficient dissipation of axisymmetric modes by the resonance absorption process.

As suggested in the discussion above, the physical models proposed thus far can be divided into two broad classes; (1) models which incorporate dissipation of the p-mode energy within the spot (i.e. the irreversible transfer of energy into heat), and (2) dissipationless models which rely on conversion of the p-mode flux into other wave modes. Let us begin with a discussion of the dissipative models. 


\section{Dissipative Models}

Two dissipative mechanisms have been proposed. The first, resonance absorption, is an extension of a wave dissipation model developed for the corona. The second is radiative damping within the sunspot. These are discussed below.

\subsection{RESONANCE ABSORPTION}

Hollweg (1988) was the first to propose a theoretical explanation for the absorption of p-modes by sunspots in the context of a simplified, planar resonance absorption model. He found that resonance absorption could be important under certain circumstances relating to the angle of incidence of the wave and the ratio of specific heats inside and outside of the spot. But he concluded that the resonance absorption process, on the whole, probably could not explain the large amount of absorption seen observationally.

Lou (1989) investigated the resonant absorption of MHD waves in a viscous medium. The linearized MHD wave equations including both shear and compressive viscosity terms were solved numerically using a standard algorithm. He concluded that for nonaxisymmetric perturbations absorption at the Alfvenic singular layer could be quite effective in absorbing wave energy. Typical values for the absorption coefficient obtained in these models is on the order of $40-45 \%$, in reasonable agreement with the observed value.

For axisymmetric perturbations the story is quite different. Lou showed that when axisymmetric waves impinge on a perfectly cylindrical sunspot, there is no singularity in the ideal MHD equations. The result is that there is no resonance layer set up in the spot, and the dissipation is therefore drastically reduced within the sunspot. The analogous result for resonant dissipation in a low $\beta$ plasma was noted by Davila (1985). Whenever the transverse wavenumber $\left(k_{\perp}\right)$ vanishes the two transverse components of the momentum equation decouple and the solution changes character dramatically. Lou showed that to dissipate $50 \%$ of the amplitude of an incoming axisymmetric wave the viscosity within the spot would have to be of order $10^{5} \mathrm{~g}-\mathrm{cm}^{-1}-\mathrm{s}^{-1}$. This value is much larger than the classical value for the viscosity, implying turbulent processes are important. However the turbulent viscosity needed to explain the observations is roughly 100 times larger than the upper limit on the spatially averaged turbulent viscosity obtained by considering the observed width of the p-mode ridges themselves. Lou also points out that it is somewhat counter intuitive to expect a large increase in the turbulent viscosity within a sunspot where the magnetic field is presumed to inhibit convection.

Chitre and Davila (1989, in these proceedings) considered an analytic model for resonance absorption in a sunspot flux tube. The model was basically an extension of the calculation of Davila (1985) to incorporate cylindrical geometry and a finite sound speed. The absorption rate was calculated from the ideal portion of the solution by integrating the Poynting flux over the surface of the resonance layer. It was found that $50 \%$ of the incoming wave energy could be absorbed with rather modest wave amplitudes $(\approx .3-.5$ of the incoming wave) within the spot. The expression for the energy absorbed was seen to be proportional to $m^{-2}$ in rough agreement with the calculation of Lou.

It should be emphasized that the absorption rate obtained in this calculation is 
independent of the details of the dissipation mechanism (magnitude or physical process) assumed. Therefore the results are very general, and can be applied for viscous, ohmic, radiation or any other damping mechanism. The analytic example presented by Chitre and Davila assumed radiative damping, but the same absorption rate would be obtained for viscous or ohmic dissipation. There are however two important observables which do depend on the dissipation process; (1) the amplitude of the wave within the dissipation region, and (2) the thickness of the dissipation region. Estimates for these quantities have not yet been obtained.

The problem with the absorption of axisymmetric modes remains however. Chitre and Davila suggest that if one considers more realistic spot models this problem may disappear. For example if one considers a spot which is not exactly cylindrical, perhaps it is elliptic or it has a nearly circular but fluted surface it is clear that incoming waves which are axisymmetric would be coupled because of the asymmetric geometry into asymmetric internal modes within the spot. These modes could then be efficiently dissipated by the resonant absorption process. These same arguments would hold if the sunspot were not precisely vertical within the atmosphere. There have been no calculations of the strength of these effects, however it is clear that at least in principle non-axisymmetric geometry of the spot itself may be able to account for the observed absorption of symmetric waves.

In addition, preliminary analysis of twisted flux tubes indicates that the absorption of $\mathrm{m}=0$ modes is possible, even in the context of an otherwise perfectly symmetric sunspot.

\subsection{RADIATIVE DAMPING}

In a recent paper Bogdan and Knolker (1989) consider the propagation of linearized MHD waves in a uniform radiating plasma. They conclude that in the uniform limit, radiation damping is not likely to contribute significantly to the damping of p-modes within sunspots. This conclusion can be significantly modified when cross field gradients within the sunspot flux tube are accounted for (see the discussion above).

\section{Non-dissipative Models}

There have been two non-dissipative models proposed in the literature. Neither seems to be able to explain the observed absorption of p-mode flux in their present form.

\subsection{CHROMOSPHERIC WINDOW EFFECT}

Campbell and Roberts considered the effect of horizontal chromospheric magnetic fields on the reflection of p-modes. They found that the efficiency of the reflection was a function of the horizontal wavelength of the disturbance (the results of their calculation was summarized in figure 1 of that paper). They suggested that the observed absorption of p-modes could be due to enhanced leakage of the wave flux through the chromosphere. If however one examines the calculated result carefully one finds that as $\ell$ (i.e. $k_{\perp}$ ) increases more and more frequencies are trapped in the photosphere. This wavelength dependence seems to be contrary to the observed result that the sunspot absorption 
increases with increasing $k_{\perp}$.

\subsection{RESONANT SCATTERING}

Bogdan (1989) considers the scattering of plane sound waves by sunspots. He finds that the transmission coefficient has resonant peaks, suggesting increase absorption at discrete frequencies. Bogdan suggests that this effect could result in the absorption observed by Braun, Duvall and LaBonte.

It seems clear however that this mechanism alone cannot explain the absorbed pmode flux because the total energy in the system is conserved. The model must be modified to include dissipation or leakage at the boundary to obtain agreement with the observations.

\section{Conclusions}

There is no satisfactory theoretical model for the absorption of p-modes by sunspots available at this time. The resonance absorption model seems to be able to obtain the large observed absorption coefficients observed for non-axisymmetric perturbations. For axisymmetric perturbations, departures from perfect cylindrical symmetry or the inclusion of a slight twist in the sunspot flux tube may be able to resolve the problem with the absorption of $m=0$ wave modes. A calculation of the magnitude of these effects is not available at this time. Other dissipative models, which do not incorporate the background gradient effects inherent in the resonance absorption mechanism, require uncomfortably large dissipation coefficients within the sunspot.

The chromospheric window effect seems to have a wavelength dependence which is contrary to observation, and the resonant scattering effect proposed by Bogdan cannot explain the observed p-mode absorption without the addition of an energy loss mechanism to the model.

Acknowledgement: The author wishes to Drs. S. M. Chitre and T. L. Duvall for constructive comments on the initial draft of this paper.

\section{References}

Abdelatif, B. W., B. W. Lites, and J. H. Thomas, 1986, Ap. J., 311, 1015.

Bogdan, T. J., 1989, Ap. J., 345, 1042.

Bogdan, T. J. and M. Knolker, 1989, Ap. J., 339, 579.

Braun, D. C., T. L. Duvall, and B. J. LaBonte, 1987, Ap. J., 319, L27.

Braun, D. C., T. L. Duvall, and B. J. LaBonte, 1988, Ap. J., 335, 1015.

Campbell, W. R. and B. Roberts, 1989, Ap. J., 338, 538.

Davila, J. M., 1985, Ap. J., 291, 328.

Duvall, T. L., 1982, Nature, 300, 242. 
Hollweg, J. V., 1988, Ap. J., 335, 1005.

Lou, Y., 1989, Viscous Magnetohydrodynamic Modes and p-Mode Absorption by Sunspots, in press.

Thomas, J. H., L. E. Cram, and A. H. Nye, 1982, Nature, 297, 485.

\section{DISCUSSION}

PRIEST: (i) What is the wavelength of the observed modes compared with the spot radius?

(ii) If a wavetrain is locally incident from one direction rather than from all directions, why should the non-axisymmetric modes not be more important than the axisymmetric ones?

(iii) What has been learnt so far from sunspot seismology about the subsurface nature of sunspots?

DAVILA: (i) A range of horizontal wavelengths is observed but typically the wavelengths observed are on the order of the spot diameter.

(ii) The p-modes in the vicinity of the spot are assumed to be nearly isotropic. For that reason the velocity expansion in terms of cylindrical waves is probably more appropriate than the plane wave expansion that you suggest.

(iii) We have learned quite a lot observationally about the interaction of p-modes and sunspots. Converting this information into inferences regarding the physical nature of the sunspot below the surface, one must have a theory for the interaction. A generally accepted theory does not exist at this time.

ROBERTS: I think it may be important that a spot has both a vertical structure (the umbra) and a horizontal one (in the penumbra). So two effects may be at work in the absorption of p-modes, one due to the vertical plug of field and the other due to the horizontal penumbral canopy. Is there any evidence for two aspects in the data of Braun et al.?

DAVILA: The absorption seen in the observations is clearly not confined to the umbra of the spot. Typically absorption seems to occur in a region 2-3 times larger than the sunspot umbra.

MOGILEVSKIJ: We have measured the spectrum of oscillations in sunspot umbra and found that in the low sound spectrum there are some discrete absorption lines with the frequences $\omega=n \omega_{p}(n=1,2, \ldots)$. These lines give information on subphotospheric dynamics down to $10^{9} \mathrm{~cm}$.

UBEROI: (i) Why do you rule out the additional singularity arising due to compressibility for the $\mathrm{m}=0$ mode?

(ii) What are the magnetic field ranges in which sound wave conversion takes place?

DAVILA: (i) Any singularity in the equation can in principle provide a site for dissipation in the plasma. 
(ii) p-modes are absorbed in regions with surface field strengths which range from 100 to a few thousand Gauss.

HOLLWEG: (i) In response to Dr Uberoi's question, I have looked at the cusp singularity and concluded that it exists only under very extreme parameters. (ii) If the spot "surface" is corrugated, it loses its meaning and the m-difficulties go away. The talk by Nigel Weiss suggests evidence for fluting so we may indeed have corrugation.

DAVILA: (ii) The coupling between $\mathrm{m}=0$ and $\mathrm{m}>0$ modes caused by surface corrugations on the sunspot flux tube will have a strength proportional to the amplitude of the corrugation We need to calculate what amplitude is necessary to provide the observed coupling. The initial sunspots analyzed by Braun, Duvall and La Bonte were chosen for their circular symmetry. Of course the sub-surface structure of the tube is not directly observed. 